\title{
NECROLÓGICO
}

\section{A OBRA CIENTÍFICA DE ANTENOR LEITÃO DE CARVALHO (1910-1985)}

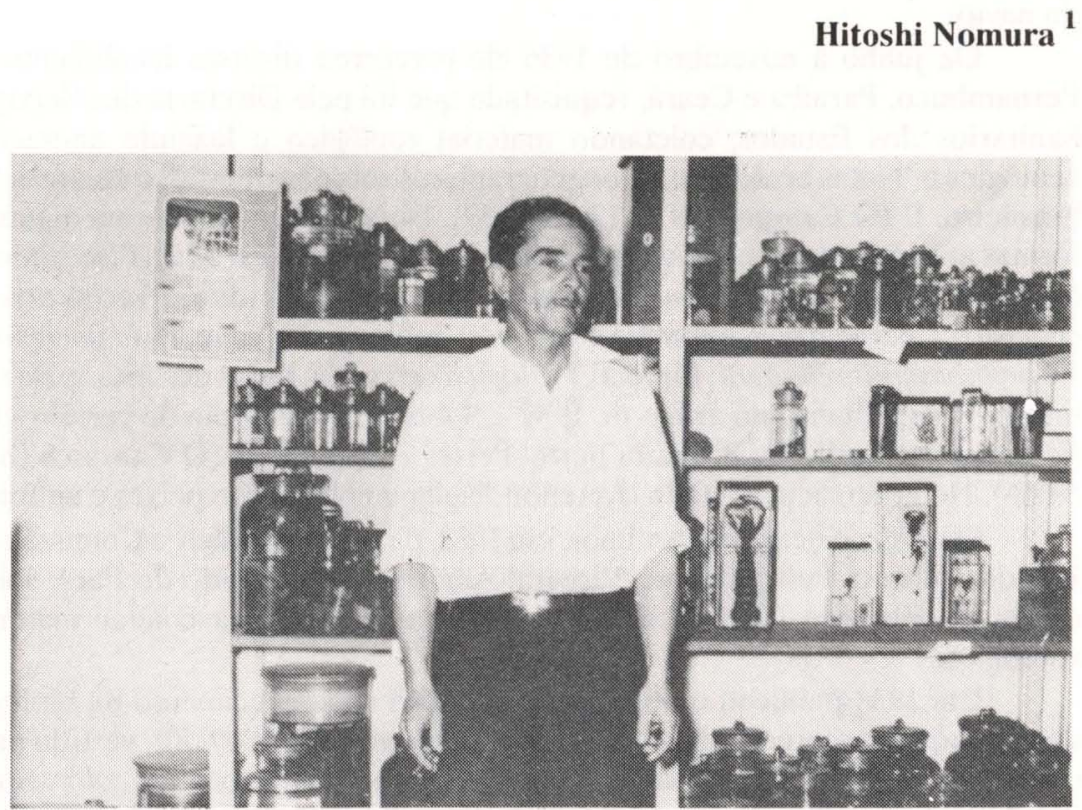

Antenor Leitão de Carvalho fotografado por Herbert R. Axelrod em seu laboratório no Museu Nacional do Rio de Janeiro.

Antenor Leitão de Carvalho era natural de Barreira do Piquete, São Paulo, onde nasceu no dia 15 de abril de 1910, tendo falecido na cidade do Rio de Janeiro, Rio de Janeiro, em 11 de dezembro de 1985.

Desconhecemos suas atividades anteriores a 1927. Sabemos que de 1927 a 1932 foi piloto da Marinha Mercante e que em 11 de janeiro de 1933 resolveu se inscrever como praticante gratuito da Secção de Zoologia do Museu Nacional, cujo chefe, Alípio de Miranda Ribeiro, o introduziu no mundo da ictiologia e da herpetologia. Produziu alguns trabalhos de ictiologia, mas Antenor é mais conhecido pelos seus artigos sobre anfíbios.

Antenor não tinha formação acadêmica como acontecia com muitos zoólogos da sua época, mas tornou-se um grande pesquisador, graças aos seus

1) Escola Superior de Agronomia Luiz de Queiroz, 13418-900 Piracicaba, São Paulo, Brasil. 
esforços e aos dois orientadores que teve: Alípio de Miranda Ribeiro (18741939) e George Sprague Myers (1905-1985). Era também um excelente desenhista, como se pode notar pelas ilustrações que acompanham os seus trabalhos.

Em abril de 1935, num porto do Rio Grande do Sul, Antenor fez observações "Sobre a maneira de dispersão de um pequeno Coleóptero (Curculionidae)" (Bol. Mus. Nac. 11 (3/4): 104-105), trazido pelo vento nordeste por meio de filamentos brancos, que ficaram aderidos aos mastros, estais e varandas do navio.

De junho a novembro de 1936 ele percorreu diversas localidades de Pernambuco, Paraíba e Ceará, requisitado que foi pela Diretoria dos Serviços Sanitários dos Estados, coletando material zoológico e fazendo anotações ecológicas: "Notas ecologicas e zoogeographicas sobre vertebrados do nordeste brasileiro. I" (O Campo 8 (87): 12-15, 1937). Dessa coleta resultaram algumas formas até então desconhecidas pela ciência: peixes - Loricariidae, Plecostomus carvalhoi, hoje Hypostomus carvalhoi; Trachycorystidae, Trachycorystes cratensis; Characidae, Curimatus notonotos; anfíbios - Hylidae, Hyla pachyurus e Corythomantis schubarti; Pipidae, Protopipa carvalhoi, todas descritas pelo seu mestre A.M. Ribeiro no artigo de 1937 - "Sobre uma collecção de vertebrados do nordeste brasileiro. Primeira parte: Peixes e batrachios" (O Campo 8 (85): 54-56). No artigo acima citado, Antenor divulgou notas sobre peixes e anfíbios.

O governo designou Antenor, em 1937, para acompanhar a Comissão de Estudos sobre a Leishmaniose Visceral Americana no Estado do Pará, sob a chefia de Evandro Chagas. Ele aproveitou a viagem para coletar material zoológico.

Em 1938 publicou o artigo "Contribuição ao conhecimento da biologia de um pequeno morcego da Amazonia" (O Campo 9 (103): 69-70), vertido para o alemão em 1940: "Zur Biologie einer Fledermaus (Thyroptera tricolor Spix) des Amazonas" (Sitz. Gesel. Natur. Freund. z. Berlin: 249-252). Essa Espécie habita as prefoliações da musácea sororoca, Ravenala guianensis e da sororoquinha, Heliconia brasiliensis, nas imediações da cidade de Abaeté, Pará.

Em 1939 foi nomeado ajudante técnico de terceira classe da Secção de Zoologia do Museu Nacional. Na qualidade de zoólogo integrou uma expedição etnográfica que explorou as margens dos rios Araguaia e Tapirapé. Os diplópodos que ele coletou foram examinados por Otto Schubart em 1947 (Bol. Mus. Nac., n.s. Zool. (82): 1-106), que descreveu algumas espécies novas; os opiliões foram estudados por C. Mello-Leitão em 1941 (Rev. Bras. Biol 1 (4): 435-442), que descreveu três gêneros e 14 espécies novas.

Ainda em 1939 Antenor escreveu "Notas sobre Hemipipa carvalhoi Mir. Rib. (Batrachia: Anura: Pipidae. Primeira parte)"(Bol. Biol.,n.s., 4 (3): 393-414) - notas biológicas. Publicou também uma "Nota previa sobre os habitos de uma interessante "perereca" bromelicola do litoral SE brasileiro (Aparasphenodon brunoi Mir.-Rib., 1920)" (O Campo 10 (116): 25-26). Em outubro de 1938 ele esteve em Mato Grosso junto com uma expedição de Lauro Travassos e colecionou batráquios, publicando em 1939: "XIV - Observações e lista dos batráquios" 
(Bol. Biol., n.s., 4 (2): 279-280).

Em 1940 publicou "Observações sobre casos de Batracofagia entre as aves" (Mem. Inst. Osw. Cruz 35 (3): 575-576), tendo encontrado restos dos anfíbios Elachistocleis ovalis bicolor (Engystomatidae) e Bufo granulosus (Bufonidae) no estômago da curicaca, Theristicus caudatus caudatus. Nesse ano foi nomeado naturalista-auxiliar XVI do Museu Nacional e participou de trabalhos no interior do Pará, colecionando material zoológico e etnográfico.

Foi nomeado naturalista XVII do Museu Nacional em 1941. Neste mesmo ano incursionou novamente pelos vertebrados, publicando "Notas sôbre os gêneros Corythomantis Boulenger e Aparasphenodon Miranda Ribeiro Amphibia, Anura, Hylidae)" (Pap. Avs. Dep. Zool. 1 (14): 101-110) e "Nota prévia sôbre uma nova espécie de "Peripatus" do Brasil Central" (Rev. Bras. Biol. 1 (4): 447-448), ampliando este estudo em 1942, no artigo: "Sôbre "Peripatus heloisae", do Brasil Central" (Bol. Mus. Nac., n.s., Zool. 2: 57-111), resultado de uma coleta feita às margens do Rio Tapajós.

Em 1942 ele teve a oportunidade de percorrer a bacia do Rio São Francisco, estudando a fauna herpetológica. Esteve também no Espírito Santo, colecionando material ictiológico e herpetológico, como assistente de campo de George Sprague Myers (1905-1985), da Universidade de Stanford, Califórnia. Percorreu então o estado de Minas Gerais, colecionando material ictiológico e herpetológico em 1943 e, em 1944 os estados de São Paulo, Paraná, Santa Catarina e Rio Grande do Sul.

Antenor prestou concurso para o cargo de Naturalista do Ministério da Educação e Saúde, sendo aprovado e nomeado Naturalista Classe "J" em 18 de julho de 1944, lotado na divisão de Zoologia do Museu Nacional. Em 1945 publicou com Myers, "Notes on some new and little-known Brazilian amphibians, with an examination of the history of the Plata salamander, Ensatina platensis" (Bol. Mus. Nac., n.s.. Zool. (35): 1-37), tendo descrito a nova espécie de sapo Bufo dapsilis (Bufonidae). Ainda em 1944 trabalhou no Pará, no Serviço de Biologia da Pesca, continuando na mesma atividade em 1945, no Ceará. Novamente com Myers publicou o artigo: "A strange new leaf-nosed lizard of the genus Anolis from Amazonia" (Bol. Mus. Nac., n.s. Zool. 43: 1-22), descrevendo A. phyllorhinus.

Juntamente com J.R. Bailey, da Duke University, Antenor descreveu a espécie nova Leptotyphlops cupinensis em: "A new Leptotyphlops from Mato Grosso, with notes on Leptotyphlops tenella Klauber" (Bol. Mus. Nac., n.s., Zool. 52: 1-7), por ele capturada no nordeste de Mato Grosso em 1940. Ainda no mesmo ano ele descreveu "Um novo gênero de Ceratofrididae (Amphibia) do sudeste baiano - Amphibia, Anura, Ceratophrydidae" (Bol. Mus. Nac., n.s., Zool. 73: 1-18) - gênero Macrogenioglottus, dando à espécie o nome de alipioi, em homenagem ao seu primeiro orientador científico.

Por merecimento, Carvalho foi promovido a Naturalista classe "K" em 1947 e contemplado com uma bolsa de estudos da John Simon Guggenheim Memorial Foudation. Durante todo o governo de Dutra foi-lhe negada per- 
missão para se ausentar do país.

Em 1948 ele teceu considerações "Sôbre a validez de Stereocyclops incrassatus Cope, 1871 e Hypopachus mülleri (Boettger), 1885 - Amphibia - Anura - Microhylidae - Microhylinae" (Bol. Mus. nac., n.s., Zool. 84: 1-23), e com J.R. Bailey publicou: "Sôbre os hábitos e ecologia de "Pleurodema diplolistris" (Peters) (Amphibia Anura) (Rev. Bras. Biol. 8 (2): 261-264).

Preparou "Notas sôbre os hábitos de "Dendrophryniscus brevipollicatus" Espada (Amphibia, Anura)", publicando-as em 1949 (Rev. Bras. Biol. 9 (2): 223-227). Ainda em 1949 foi requisitado pelo Instituto Nacional do Pinho, tendo permanecido de abril a junho na Estação Florestal dos Pardos, em Três Barras, município de Canoinhas, Santa Catarina, estudando o comportamento dos animais em relação às árvores de valor econômico como o pinheiro, a imbaúba, o cedro e outras: "Contribuição ao estudo da biologia na Estação Florestal dos Pardos" (Anuár. Bras. Econ. Flor. 3 (3): 208-222), publicado em 1950. No pinheiro ele observou lagartas da mariposa Laspeyresia sp., tanto em suas folhas quanto nos galhos. Verificou seu encasulamento e posterior surgimento do adulto. Observou também a presença de trips, Hoplandrothrips sp., atacando as suturas das folhas dos botões terminais; coccídios (Diaspididae); formigas quem-quem, Acromyrmex crassipinus e mineira, A. multicinodus. A perdiz, Rhynchotus $r$. rufescens, come os brotos do pinheiro, assim como arranca a semente para comer a raiz da nova planta. Viu grandes bandos de psitacídeos, Amazona vinacea, Pionus maximiliani siy e Pyrrhura f. frontalis, alimentando-se de pinhões. Já o serelepe, Sciurus ingrami, o ratinho-do-mato, Oligoryzomys utiaritensis e o ouriço, Coendou roberti, são acusados de arrancar os pinhões das covas para comê-los. A cutia, Dasyprocta azarae catrinae, é apreciadora de pinhões e costuma enterrá-los. Também se alimentam de pinhões a queixada, Tayassu pecari e o cateto, T. tajacu. No cedro ele estudou uma broca, Hypsipyla grandella, cujas lagartas perfuram o gomo vegetativo da plantinha, destruindo sua medula; observou o pulgão Freysuila sp. atacando os galhos e folhas. Costa Lima, que analisou os insetos, achava que entre eles havia espécies novas.

Carvalho, em 1950, foi novamente requisitado pelo Instituto Nacional do Pinho, para estudar a biologia dos insetos nocivos às essências florestais de São Paulo e Paraná. Trabalhou também no Brasil Central, na região dos Rios Culuene e Xingu, colecionando material zoológico.

As "Observações sobre "Tropidophis paucisquamis" (Müller, 1901)" (Rev. Bras. Biol. 11 (3): 239-248) - publicado em 1951 - o menor dos boídeos brasileiros. Os dois exemplares que ele observou foram capturados na Serra do Mar em Angra dos Reis, Rio de Janeiro, em 28 de março de 1948. É uma espécie que ingere batráquios.

Carvalho finalmente obteve permissão para se ausentar do País em 1951, para usufruir a bolsa de estudos que lhe foi concedida pela Fundação Guggenheim em 1947. Dirigiu-se para o Museu de História Natural da Universidade da Califórnia, onde trabalhou sob a orientação de George Sprague Myers. Lá ficou até meados de 1953, por haver obtido renovação da bolsa. Então aprovei- 
tou para cursar as cadeiras de Herpetologia, Ictiologia e Anatomia Comparada oferecidas por aquela Universidade, assim ampliando seus conhecimentos acadêmicos. Durante sua permanência nos Estados Unidos publicou em 1952, com Myers, "A New Dwarf Toad from Southeastern Brazil" (Zoologica 37 (1): 1-3) sobre o sapo anão, Bufo pygmaeus, espécie nova. Com J.M. Savage publicou em 1953 "The Family Position of Neotropical Frogs Currently Referred to the Genus Pseudis" (Zoologica 38 (4): 193-200, 1953), mostrando a posição de Pseudis dentro das famílias das rãs neotropicais. Aceitando uma sugestão de Myers, publicou em 1954 "A preliminary synopsis of the genera of American Microhylid frogs" (Occas. Pap. Mus. Zool. Univ. Michigan (555): 1-20), quando criou os gêneros Hyophryne, Hamptophryne (separando-o do gênero Chiasmocleis), Arcovomer e a espécie. A. passarellii, Relictivomer, Myersiella e Synapturanus.

Em 1955 Antenor teve a oportunidade de acompanhar a expedição conjunta do Museu de Los Angeles com o Museu Nacional ao interior de Goiás. Nesse ano publicou um excelente estudo sobre "Os jacarés do Brasil" (Arq. Mus. Nac. 42 (1): 127-151), descrevendo as seis espécies distribuídas por três gêneros, tendo recebido sugestões de J.R. Bailey, da Universidade de Michigan, com quem trabalhara em 1946.

No artigo de Myers de 1955, "Notes on the Classification and Names of Cyprinodont Fishes" (The Tropical Fish Magazine 4 (7): 7), se encontra a descrição sumária da nova espécie Potamophylax pygmaeus Myers \& Carvalho, válida frente às regras de nomenclatura zoológica. O gênero Potamophylax é o nome de um inseto neuróptero criado por Wallengren em 1941. Por isso, Tyson R. Roberts ("Description, osteology and relationships of the Amazonian Cyprinodont fish Fluviphylax pygmaeus (Myers and Carvalho)" (Breviora. Mus. Comp. Zool. (347): 1-28 - 1970), propôs a mudança do gênero para Fluviphylax Roberts, 1970 e fez uma redescrição completa da espécie $F$. pygmaeus (Meyers \& Carvalho).

Em 1957 Carvalho foi designado para exercer a função de Chefe da Divisão de Zoologia do Museu Nacional, na qual permaneceu até junho de 1961. Ainda em 1957 passou a publicar "Notas para o conhecimento da biologia dos peixes anuais" (Rev. Bras. Biol. 17 (4): 459-466). Esses peixes vivem em poças temporárias de água, sendo chamados "peixes das nuvens" porque o povo pensa que eles são trazidos pelas nuvens e chuvas. Nesse primeiro artigo ele descreveu os hábitos de Cynolebias whitei, reproduzido em 1966 em O Aquarista 3 (8): 19-24 e em inglês (setembro de 1958) sob o título "The Fish That Spawns Underground" (Tropical Fish Hobbyist 7 (1): 16-26). Ele voltou ao assunto dos peixes anuais em 1959, ao descrever um "Novo gênero e nova espécie de peixe anual de Brasília, com uma nota sobre os peixes anuais da baixada fluminense, Brasil (Pisces, Cyprinodontidae, Fundulinae)" (Bol. Mus. Nac., n.s., Zool. (201): 1-20), reproduzido, em 1966, em O Aquarista 3 (6/7): 37-38).

Com Bertha Lutz, em 1958, descreveu um novo gênero e uma nova espécie de anfíbio anuro Paratelmatobius lutzi: "Novos Anfíbios Anuros das Serras Costeiras do Brasil (Mem. Inst. Osw. Cruz 56 (1): 239-249). 
Em 1959 descreveu, com Myers novo gênero e espécie de peixe da família Characidae, Sartor respectus, do alto Rio Xingu em "A Remarkable New Genus of Anostomin Characid Fishes from the Upper Rio Xingú in Central Brazil" (Copeia (2): 149-152).

Em 1960 participou do I Congresso Brasileiro de Zoologia, para o qual enviou três trabalhos, inéditos até hoje: "Inventário da Fauna do Brasil", "Os Onicóforos" e "As Cecílias e as salamandras".

Em 1961 Carvalho participou da lista tríplice para a escolha do Diretor do Museu Nacional, período de 1961-1964. Em 1963, a convite do Departamento de Biologia da University of Southern California, tomou parte do Seminário de Biologia Tropical I, patrocinado por essa Universidade e pela National Science Foundation, realizado na Universidade de Costa Rica, em San José. Foi eleito Vice-Diretor do Museu Nacional em 1964, para o período 1964-1967 e, no mesmo ano, designado Diretor da Divisão de Zoologia (1964-1967).

Antenor voltou a tratar dos anfíbios em 1965, discutindo a validade do emprego de caracteres larvais como principal índice filético em Amphibia Salientia, tendo M.I. Griffiths, do Birkbeck College - University of London, como co-autor em "On the validity of employing larval characters as major phyletic indices in Amphibia, Salientia" (Rev. Bras. Biol. 25 (2): 113-121).

Com M.A. Freiberg em 1965, tratou do "El yacare sudamericano Caiman latirostris (Daudin)" (Physis, Buenos Aires, 25 (70): 351-360), de ampla distribuição geográfica, desde o Rio Grande do Norte até o Rio Paraná na Argentina e também no Paraguai e Uruguai. Devido às diferentes condições ecológicas, a espécie se diversificou e por isso os autores propuseram os nomes de C.l. latirostris para a subespécie típica e $C$. l. chaconensis, habitante das províncias de Entre Rios, Santa Fé, Chaco, Formosa e Jujuy.

Em 1966 Antenor publicou "Novos dados para o conhecimento de "Phreatobius cisternarum" Goeldi (Pisces, Pygidiidae, Phreatobiinae)" (Atas Simp. Biota Amaz.(Limn.) 3: 83-88).

Em 1971 descreveu "Um novo peixe anual do Estado do Espírito Santo (Pisces, Cyprinodontidae, Rivulinae)" (Rev. Bras. Biol. 31 (3): 401-404, 1971), crismando-o de Cynolebias myersi, em homenagem ao seu orientador, como sua contribuição ao "Festchrift for George Sprague Myers", promovido pelos exalunos desse mestre, para comemorar a sua aposentadoria em 1970.

Durante o III Congresso Brasileiro de Zoologia ele foi Presidente da Secção I - Vertebrados, em 1968, no Rio de Janeiro.

Em 1971 Antenor foi designado Chefe do Departamento de Vertebrados (1971-1973), reeleito em 1973 (1973-1975) e em 1975 (1975-1977) e, em 1977, chefe-substituto (1977-1979).

Carvalho participou de diversas bancas examinadoras de candidatos ao título de Mestre em Zoologia na Universidade Federal Rural do Rio de Janeiro: Sila Tenório de Albuquerque em 1976, Oswaldo Luiz Peixoto (como orientador) e José Vanderli Andreata (como orientador) em 1977, Carlos Alberto Gonçalves da Cruz (como orientador) em 1978, Sueli Pontes de Fábio, Aída Maria 
Eschinazi de Oliveira, José Luiz Helmer (como orientador) em 1979, Sérgio Furtado dos Reis e como orientador de Ivani Callado de Souza (como orientador) e João Farra Muro (como orientador) em 1980. Em 1978 foi membro efetivo da comissão examinadora do concurso público para Professor Assistente de Zoologia Geral do Instituto de Biologia da Universidade Federal Rural do Rio de Janeiro.

No período de 1975-1979 lecionou Herpetologia no curso de Pós-graduação em Zoologia dessa Universidade, juntamente com Eugênio Izecksohn.

Antenor descreveu em 1985, junto com seu orientado C.A.G. Cruz, uma nove espécie de peixe anual "Um Novo Cynolebias do Sudeste Baiano (Pisces, Cyprinodontidae, Rivulinae)" (Arq. Univ. Fed. Rur. Rio de Jan. 8 (1/2): 11-15), Cynolebias bokermanni, homenageando o conhecido herpetologista C.A. Werner Bokermann. Foi seu último artigo científico, completando 50 anos de publicações (1935-1985).

Antenor Leitão de Carvalho foi sócio da Sociedade de Biologia do Rio de Janeiro, da American Society of Icthyologists and Herpetologists, da Asociación Latino-Americana de Ictiologos y Herpetologos, da The Herpetologist's League, da Society for the Study of Amphibians and Reptiles e Full Member da Society of the Sigma XI Stanford Chapter.

Acabamos de ver que Antenor publicou a maior parte dos seus artigos concentrando-se nos anfíbios, depois nos peixes anuais, répteis (cobra, lagarto e jacarés) e insetos (besouros e mariposas).

Segundo alguns zoólogos, Antenor era reservado e taciturno. Jorge Jim, da Universidade Estadual Paulista de Botucatu, São Paulo, escreveu-nos que ele "Era possuidor de um especial dom de comunicação; contava as histórias, muitas vezes em tom anedótico, de suas aventuras".

Sabemos que ele possuía dados sobre quelônios e outros peixes anuais, que ainda permanecem inéditos. Alguns dos cágados ainda estavam vivos (1990) e são espécies não descritas; Antenor apenas aguardava a morte natural deles, para então considerá-los como tipos para descrição científica. Algum sucessor seu provavelmente fará essa descrição.

Antenor Leitão de Carvalho foi homenageado pelos seus colegas de profissão e seu nome consta num gênero e várias espécies - gênero de DIPLOPODA - Spirostreptidae: Carvalhodesmus Schubart, 1945; espécies de: AMPHIBIA - Pipidae: Protopipa carvalhoi Ribeiro, 1973;

Ceratophrydidae: Odontophrynus carvalhoi Savage \& Cei, 1965; Microhylidae: Syncope antenori Walker, 1973, Syncope carvalhoi Nelson, 1975; Hylidae: Hyla carvalhoi Peixoto, 1981; Leptodactylidae: Zachaenus carvalhoi Izecksohn, 1982; PISCES - Loricariidae: Plecostomus carvalhoi Ribeiro, 1937; Aspredinidae: Bunocephalus carvalhoi Ribeiro, 1944; Cyprinodontidae: Cynolebias carvalhoi Myers, 1947; Cyprinodontidae: Cynolebias antenori Myers, 1952; DiPloPODA - Spirostreptidae: Stenostreptus carvalhoi Schubart, 1945; Vanhoeffeniidae: Arndtodesmus carvalhoi Schubart, 1947; ARANEAE Argiopidae: Mecynogea carvalhoi Melo Leitão, 1944, Micrathena carvalhoi Melo 
Leitão, 1944; SCORPIONIDA - Buthidae: Tityus carvalhoi Melo Leitão, 1945; OPILIONES - Gonyleptidae: Discocystus carvalhoi Melo Leitão, 1941; NEMATODA - Icthyocephalidae: Icthyocephalus antenori Travassos \& Kloss, 1959.

AGRADECIMENTOS. Somos gratos ao Dr. Eugênio Izecksohn pelo fornecimento do curriculum vitae de A.L. Carvalho; ao Dr. Melquíades Pinto Paiva por alguns dados pessoais e ao Dr. Werner Bokermann pelas xerocópias de vários artigos de Carvalho.

Recebido em 30.XII.1991; aceito em 25.XI.1993. 